\title{
La protection anti-corrosive de la conduite forcée de Montpezat
}

\section{Protecting the Montpezat penstock against corrosion}

\author{
PAR M. CROYERE
}

DIRECTEUR TECHNIQUE DE LA SOCUÉTÉ BITULAC

\begin{abstract}
Problème de la protection anticorrosive d'une conduite blindée dans le béton.

La protection devait être particulièrement soignée du fait de la nature des eaux, et surtout du fait que cette protection ne pouvait être entretenue.

Il a été choisi un revêtement d'émail bitumineux appliqué à chaud.

L'auteur fait des remarques sur la façon dont doit être conçu un tel chantier.
\end{abstract}

\begin{abstract}
The problem of protecting a lined pipe in concrete.

The protective material had to be applied very carefully because of the nature of the water and above all because maintenance work could not be carried out on it.

A facing of Bituminous Enamel to be applied when hot, was selected.

The author comments on how such a work site should be planned.
\end{abstract}

L'ouvrage de Montpezat est la réalisation d'un projet assez audacieux qui consiste à collecter les eaux du bassin versant de la Loire supérieure, comprenant les rivières de la Loire, du Gage et de la Veyradière et à les déverser dans l'Ardèche, c'est-à-dire dans le versant du Rhône.

Cet ouvrage, tout en galeries souterraines, se termine sur une chute de $625 \mathrm{~m}$ par une conduite forcée de $1.473 \mathrm{~m}$ de longueur et de $2,30 \mathrm{~m}$ de diamètre, précédée d'une galerie de $2,60 \mathrm{~m}$ de diamètre, blindée sur $600 \mathrm{~m}$ de longueur.

Le site de Montpezat a été choisi comme le plus favorable pour y placer la centrale, mais, afin de profiter de la plus grande hauteur de chute possible, cette centrale a été construite en souterrain à $130 \mathrm{~m}$ de profondeur environ par rapport au poste extérieur. Cette disposition a orienté la conception de la couduite forcée qui, elle aussi, comme tout l'ouvrage, a été creusée dans la roche dont est formée la montagne.
La partie métallique a protéger contre la corrosion comprend :

- Une galerie blindée horizontale de $600 \mathrm{~m}$ de longueur; diamètre : $2,60 \mathrm{~m}$; épaisseur de la tôle : $12 \mathrm{~mm}$;

- Une cheminée d'équilibre de $111 \mathrm{~m}$ de hauteur et de $3,50 \mathrm{~m}$ de diamètre;

- Une conduite forcée de $1.473 \mathrm{~m}$ de longueur avec une pente de $46 \%$ et un diamètre de $2,30 \mathrm{~m}$; épaisseur de la tôle : 12 à $35 \mathrm{~mm}$;

- Un collecteur sur construction soudée.

Cet important ouvrage souterrain est seulement accessible par trois endroits :

- Le trou d'homme de l'extrémité aval, dans l'usine;

- La fenêtre F 4, à mi-hauteur de la conduite forcée, à peu près au milieu de la pente; 
- La fenêtre F 3, au pied de la cheminée d'équilibre, à l'entrée du troncon de galerie conduisant à la cheminée de la vanne de tête.

L'accès de cette conduite souterraine est donc très difficile du fait du petit nombre de « fenètres $\gg$, et de la pente importante imposant de travailler sur une plate-forme suspendue à plus de 600 m de câble.

\section{Données du problème}

Le bassin versant de la Loire supérieure est constitué par du terrain volcanique et présente une épaisseur d'humus négligeable. II est donc peu sujet à retenir les eaux de pluie et celles-ci sont collectées dans ses ruisseaux, dans le même état, à peu de chose près, que celui où elles se trouvaient en tombant du ciel.

Les analyses des trois sources d'eau donnent, en effet :

\begin{tabular}{|c|c|c|c|}
\hline \multicolumn{2}{|c|}{ VEYRADÊRE } & GaGE & LOIRE \\
\hline $\mathrm{pH}$ & 7,2 & 7,5 & 7 \\
\hline $\mathrm{CO}^{2}$ libre & 1,1 & 0,22 & 0,22 \\
\hline Alcalinité & $6^{\circ}$ & $5^{\circ}$ & $5^{\circ}$ \\
\hline
\end{tabular}

Ces eaux sont très peu chargées en calcaire, non incrustantes, très agressives pour le béton et relativement agressives pour le fer. Nous voulons dire par là que, sans être en mesure de provoquer des corrosions profondes et rapides, comme certaines eaux, elles provoqueront des attaques par chancres d'aération différentielle, pouvant percer $10 \mathrm{~mm}$ de tôle en quinze ou vingt ans.

Cette eau est absolument limpide et ne laisse aucun dépôt. L'analyse bactériologique n'a pas été faite, mais il semble qu'elle doit être négative, étant donné la très faible surface des retenues récentes (barrages du Gage et de la Veyradère), et la décantation prolongée subie dans le lac naturel d'Issarlès.

Etant donné la difficulté considérable que représenterait une réparation de conduite dans cette situation souterraine, il fallait mettre absolument à l'abri de toute corrosion ce tube d'acier soudé au rocher par une liaison en béton.

On avait à choisir entre plusieurs techniques :

- Soit l'utilisation de peintures minces;

- Soit l'utilisation de ces mèmes peintures sur un fond de métallisation au zinc;

- Soit l'utilisation d'émail bitumineux appliqué à chaud, avec ou sans protection cathodique.

A première vue, étant donné que l'on désirail

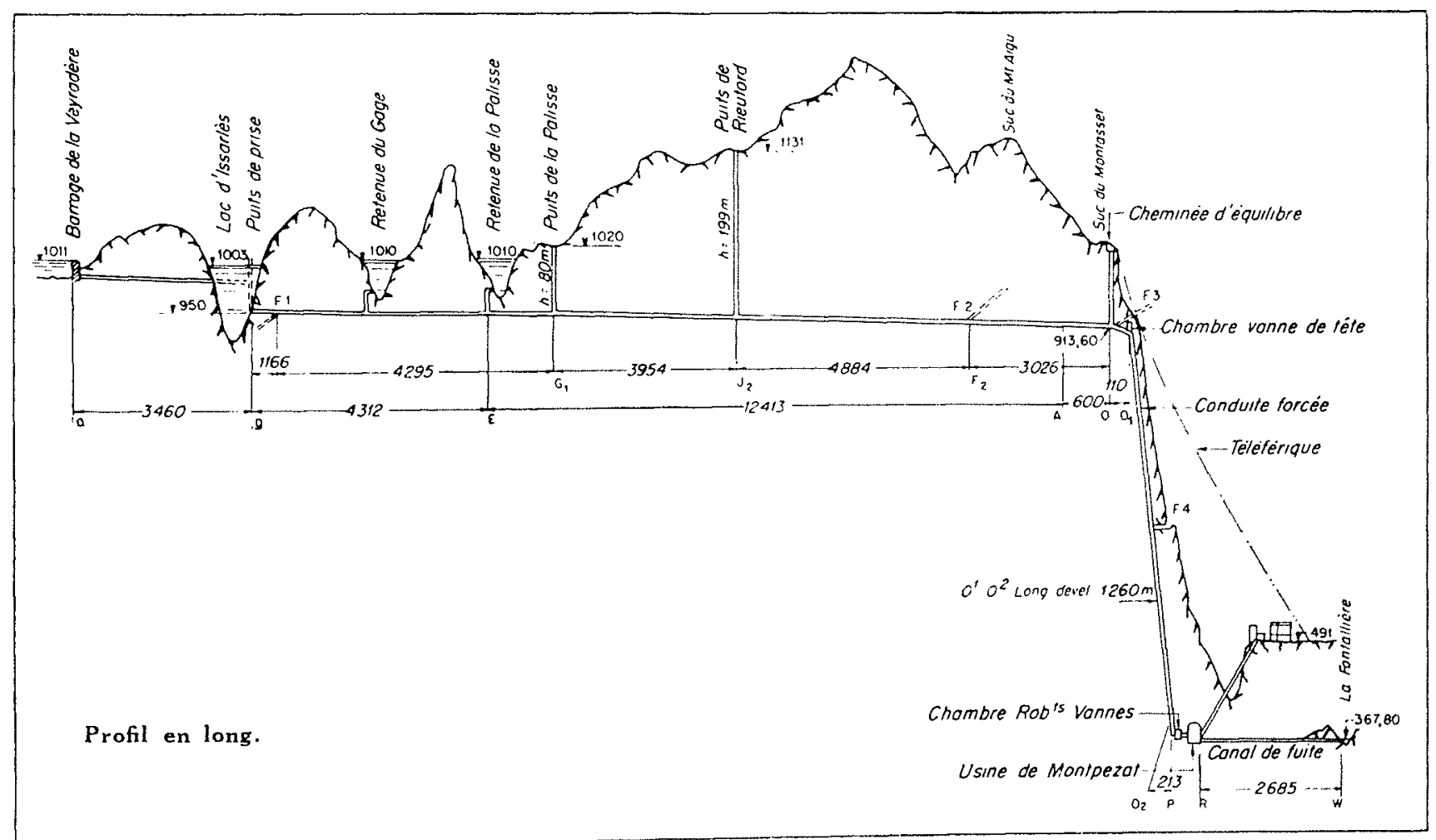


obtenir une tenue de très longue durée, c'est vers la troisième classe de protection que l'on pensait immédiatement se tourner, sans y ajouter de protection cathodique, impraticable avec des eaux si peu chargées.

Mais, appliquer un émail à chaud dans de telles conditions, n'était-ce pas aller à un échec? En effet, cette longueur de $1.473 \mathrm{~m}$ en forte pente, ne présentant qu'un seul point d'accès au milieu, permettrait-elle de mettre en œuvre une technique exigeant autant de soins que celle de l'émaillage à chaud? Ne valait-il pas mieux choisir une technique plus simple, comme celle comportant trois au quatre couches de peinture, ou la métallisation?

En fait, ce fut l'émail à chaud qui fut choisi : - D'une part parce que les eaux véhiculées sont trop agressives pour la technique - très bonne par ailleurs - de la métallisation;

- D'autre part, dans les conditions de service à Montpezat, les meilleures peintures à froid ne peuvent, sans entretien, fonctionner plus de dix ans et, après ce délai, il est vraisemblable que des chancres de rouille se seront formés. Car il est très difficile de reprendre de façon définitive la protection d'une conduite rouillée par chancres.

Il fallait un revêtement plus solide qu'une peinture et si l'on voulait réellement résoudre le problème malgré les difficultés de réalisation, il fallait appliquer un produit épais, un émail à chaud. Avec ces produits, en effet, on obtient un revêtement dont. l'épaisseur minimum est de 1,5 mm, soit l'équivalent de 20 couches de peinture.

L'émail qui fut choisi pour Montpezat avail déjà fail ses preuves dans des cas difficiles, en particulier pour la protection de conduites de vidange d'eaux agressives et animées de très grandes vitesses (conduites de décharge de Bortles-Orgues). Il s'agit d'un produit brun, com-

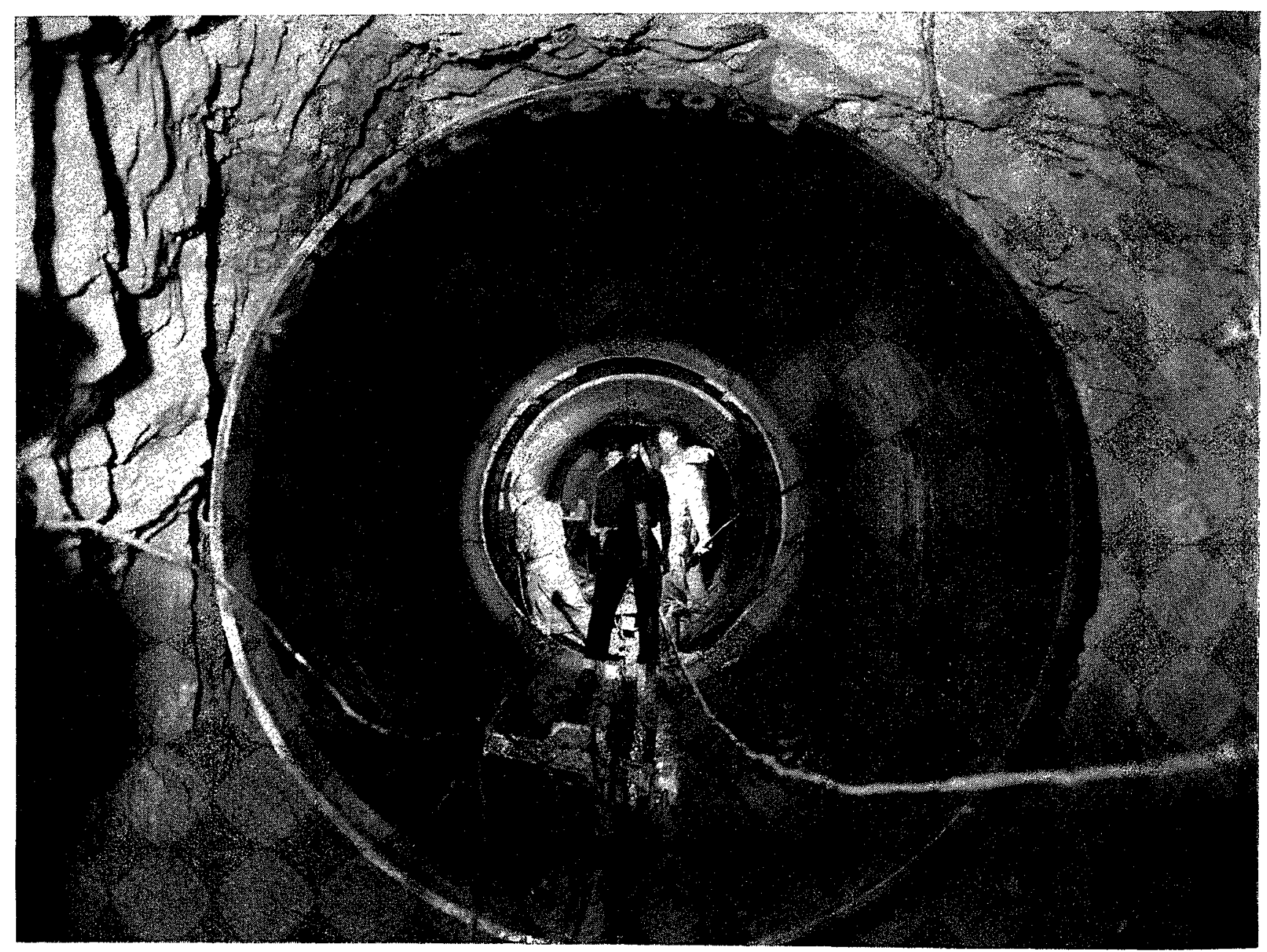

FIG. 2

Photographie illustrant le traitement que subit l'intérieur d'une conduite blindée au moment de sa soudure. 
posé d'un complexe caoutchouc-bitume, fondant $\dot{a} 180^{\circ}$ et conservant toujours une souplesse suffisante pour résister au choc et à l'abrasion.

\section{Comment l'enduit protecteur fut-il appliqué?}

Le sablage était nécessaire. Mais il était impossible de sabler la conduite en place, car son accès inférieur et la ventilation débouchaient dans la centrale. Le sable étant incompatible avec les organes délicats des alternateurs en cours de montage, on dut faire cette opération avant montage.

Par ailleurs, si l'on sablait les tubes sur place, il fallait protéger le fer aussitôt après. On choisit donc de sabler les tubes sur parc avant montage, puis de passer sur le fer une peinture d'accrochage, livrée avec l'émail à chaud.

Les tubes furent ensuite montés, mis en place, puis injectés.

Les difficultés furent nombreuses en particulier du fait que tous les corps de métiers, et notamment les ouvriers injecteurs, durent utiliser la surface de tôle vernie comme sol de travail.

En fait, il fallut refaire pratiquement un nettoyage complet et passer une nouvelle couche de peinture d'accrochage, avant de passer l'émail à chaud.

Puis l'on commença l'émaillage. L'émail était fondu dans de petits fondoirs portatifs, chauffés au propane. L'application se faisait à la brosse.

Tout alla bien dans la cheminée d'équilibre où une plate-forme suspendue à $100 \mathrm{~m}$ de câble permettait d'exécuter le travail dans de bonnes conditions. La galerie faisant suite à la cheminée ne posa pas de grands problèmes. Dans la partie en pente, un treuil installé à la fenêtre supérieure permit de descendre un chariot sur lequel étaient placés les émailleurs. Ce travail fut pénible du fait de l'exiguité de la plate-forme, sur laquelle devaient se loger deux émailleurs, le fondoir, la réserve de produit et le matériel.

Enfin, l'on mit l'ouvrage de Montpezat en eau.

Etant donné que l'on demandait à ce revêtement une protection absolue. il était entendu qu'une visite serait faite après un an de service pour obvier aux oublis ou aux imperfections. La visite de fin d'année a révélé quelques imperfections bien réparables, couvrant quelques mètres carrés seulement (sur un total de 17.000 $\mathrm{m}^{2}$ ) et auxquelles on put porter remède rapidement.

\section{Enseignements à retirer de l'expérience de Montpezat}

Vu l'importance que revèt la protection dans le cas d'une conduite blindée, nous pensons que la technique de l'émaillage à chaud est bonne, étant donné sa solidité dans le temps.

Cependant, nous pensons que la succession logique des opérations devrait être la suivante :

- Sablage et émaillage sur parc et, seulement après :

- Montage des tubes et soudure;

- Visite générale et réparations sur le radier, raccords aux soudures.

Autrement dit, les tubes seraient posés tout émaillés, le travail consécutif à la pose ne consisterait qu'en raccordements et en réparations aux parties détériorées pendant le montage.

On peut également utiliser la technique diamétralement opposée, consistant à tout sabler après la mise en place définitive. Cette technique n'est pas toujours réalisable, on l'a vu pour Montpezat, mais elle deviendra possible lorsque l'utilisation du sablage humide sera plus répandue.

En tout ćtat de cause, il faut à notre avis choisir l'une ou l'autre de ces techniques, l'expérience faite à Montpezat d'une technique mixte s'étant révélée d'un prix de revient très élevé.

nesiri Recherchons pour nos services à Bruxelles, Ingénieur ayant bonne pratique en Centrales Hydro-Electriques et en lignes de transport à H.T. et susceptible d'effectuer des déplacements au Congo belge. Ecrire avec curriculum vitae détaillé, références et prétentions à :

SOCIÉTE DES FORCES HYDRO-ÉLECTRIQUES DE L'EST DE LA COLONIE

43-47, rue de Trêves, à Bruxelles 